\title{
Velocity of a propagating Taylor-vortex front
}

\author{
M. Niklas, M. Lücke, ${ }^{*}$ and H. Müller-Krumbhaar \\ Institut für Festkörperforschung, Kernforschungsanlage Jülich GmbH, Postfach 1913, D-5170 Jülich, West Germany
}

(Received 15 February 1989)

\begin{abstract}
We present finite-difference numerical solutions of the time-dependent Navier-Stokes equations for propagating Taylor-vortex fronts into unstable Couette flow. The propagation velocities found are in excellent quantitative agreement with the theoretical predictions for normal fluids. Deviations of previous investigations reported in the literature can be explained in terms of transients. Agreement is found also for front propagation in magnetic fluids, where a magnetic field modifies the front velocity.
\end{abstract}

In many nonlinear dissipative systems, spatially periodic structures grow out of a homogeneous basic state when the driving exceeds a critical value. 'Taylor-vortex flow in the annulus between concentric cylinders and convective rolls in a fluid layer heated from below (Rayleigh-Bénard system) serve as prototype examples for such dissipative structures. After the driving has increased to a supercritical value, these structures start growing from small imperfections. If these imperfections are located in some region in space, the pattern will then grow locally first and later on spread out into the stationary unstable state, still existing in the rest of the system: the situation gives rise to a front which connects the two states and moves with a stationary speed and stationary intensity profile into the homogeneous unstable state (see inset, Fig. 1).

In recent years, this phenomenon of "front propagation" (a review is given in Ref. 2) has been discussed theoretically for model systems which are simpler than the equations of motions of most real systems, ${ }^{3-5}$ but some of them are expected to become exact descriptions in the limit $\epsilon \longrightarrow 0$. For small $\epsilon$ characterizing small supercritical driving (e.g., $\epsilon=N_{\mathrm{Ra}} / N_{\mathrm{Ra}_{c}}-1, N_{\mathrm{Ra}}$ is the Rayleigh number in a Rayleigh-Bénard system, $\epsilon=N_{T} / N_{T_{c}}-1, N_{\mathrm{T}}$ is the Taylor number, proportional to the square of $N_{\mathrm{Re}}$, in$\operatorname{dex} c$ for "critical"), these models and the equations of motion of real systems can be approximated by a Ginzburg-Landau-type equation ${ }^{6-8}$

$$
\sigma_{0}^{-1} \partial_{t} A=\xi_{0}^{2} \partial_{z}^{2} A+A\left(\epsilon-|A|^{2}\right)
$$

The complex amplitude $A$ contains the slow variations of the pattern and may, e.g., in the Taylor-Couette system, be related to the radial velocity component $u$ in the middle of the gap by $u \propto N_{\mathrm{Re}}\left(A e^{i k x}\right)$. The scales $\xi_{0}$ and $\sigma_{0}$ depend on the special system under investigation and follow from linear stability analysis.

A striking fact is that, following from the analogy to the Fisher-Kolmogorov equation, ${ }^{9,10}$ Eq. (1) results in a front propagation which occurs at a unique velocity $2,3,5$

$$
v=\bar{v} \xi_{0} \sigma_{0} \sqrt{\epsilon},
$$

where $\bar{v}=2$ is independent of a special system. Since this selection principle is a general property of Eq. (1), which also coincides with the marginal stability hypothesis for Eq. (1), one expects that the prediction would apply to real systems. Numerical ${ }^{11}$ and experimental ${ }^{12}$ investiga- tions in the Rayleigh-Bénard system are in excellent quantitative agreement with Eq. (2). However, this is not the case in the Taylor-Couette system: The experimentally observed propagation velocity was about half the predicted value, ${ }^{13}$ numerical investigations are below $\bar{v}=2$ by about $10-15 \%{ }^{14}$ This discrepancy in the Taylor-Couette system is still unsolved and in view of the situation in the Rayleigh-Bénard system and the prototype character of the Taylor-Couette system is quite unsatisfying.

In the Taylor-Couette system, a propagating front may be generated by increasing the rotation rate of the inner cylinder (i.e., the Reynolds number $N_{\mathrm{Re}}$ ) instantaneously from zero to some supercritical value. Then, in the bulk

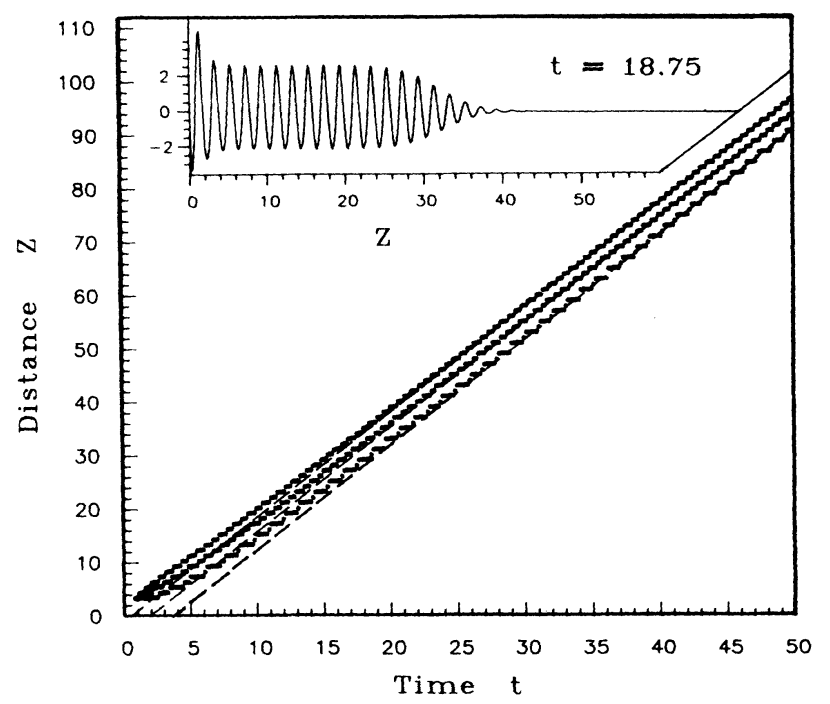

FIG. 1. Front propagation. The inset gives an impression of a propagating front by showing the radial velocity component $u(v / d)$ in the middle of the gap at a certain time $t$. The straight line on the right-hand side indicates the unstable Couette flow into which the Taylor-vortex structure is propagating at $N_{\mathrm{Re}}=1.02 N_{\mathrm{Re}_{\mathrm{c}}}$. The main figure gives the distance the intensity levels $\alpha u_{\max }(\alpha=0.1,0.25,0.5)$ of the front have traveled as a function of time. It takes a long time to approach the stationary front velocity where the dotted lines move parallel in a straight line. The dashed lines obtained by using the data for $t \geq 39\left(d^{2} / v\right)$ correspond to this velocity. [ $t$ is given in units of $\left(d^{2} / v\right) ; z$ is given in units of $(d)$.] 
of the cylinder gap, unstable Couette-flow forms first, while near the end plates of the gap (which are considered to be fixed to the outer cylinder, at rest) localized vortex flow shows up. ${ }^{15}$ These so-called Ekman vortices near the ends generate a Taylor-vortex front which propagates into the bulk of the gap. To investigate quantitative details of this front propagation, we numerically simulated rotationally symmetric flows of an incompressible fluid of kinematic viscosity $v$ in a gap of radius ratio $\eta=R_{i} / R_{0}=0.95$. The Navier-Stokes equations are solved with an explicit finite-difference method ${ }^{14,16,17}$ on a grid, spaced equally in axial and radial direction with 20 cells for the radial gap spacing $d=R_{0}-R_{i}$. It has been tested that this spacing yields results of sufficient accuracy. ${ }^{17}$ To monitor the velocity of the front, we determined positions $z_{\alpha}(t)$ where the Taylor-vortex flow has reached a fraction $\alpha=10,25$, and $50 \%$ of the maximum bulk outflow intensity $u_{\max }$ (disregarding the first maxima near the end plates). The so-defined front moves piecewise continuously, as may be seen in Fig. 1. A jump forward by approximately one vortex diameter occurs whenever the vortex ahead of $z_{\alpha}$ has grown up to $\alpha u_{\max }$. The propagation of the front can be hindered and stopped by the front, starting from the opposite end of the cylinder gap. This can be postponed by choosing a gap with a large aspect ratio $\Gamma=$ length of the gap/d. But then spontaneous nucleation of Taylor vortices will occur in the bulk of the gap resulting from fluctuations and symmetry-breaking perturbations that are always present in a real experiment. This competing growth mode of vortices seems to limit the observation time ${ }^{15}$ of front propagation in real experiments. ${ }^{13}$

However, in a numerical experiment the abovementioned disturbances can be suppressed completely. Numerical simulations allows for investigation of an ideal system which only exhibits the attributes desired- an unstable Couette flow in the bulk and some nucleus for the Taylor vortex front. Here we take $\Gamma=160$ and mirror boundary conditions at the right end of the computational mesh. The last choice significantly postpones the generation of the counter-moving vortex front. ${ }^{18}$

It takes a long time until the front becomes stationary. Figure 1 shows at the beginning distinct deviations from the front velocity observed at later times, where the three positions of the front move parallel to each other on straight lines. The final velocity is approached quickest by the $10 \%$ position line (upper line in Fig. 1) and slowest by the $50 \%$ one (lowest line in Fig. 1). Thus, experimentalists should use the smallest possible intensity value for the determination of the front velocity, a hint already given in Ref. 15. From a least-squares fit to the data in the time interval 39-50( $\left.d^{2} / v\right)$, together with values for $\sigma_{0}$ and $\xi_{0}$, which are determined with high precision ${ }^{17,19}$ by a shooting method, ${ }^{19}$ one obtains, according to Eq. (2), a dimensionless front velocity $\bar{v}=1.953$. The error, due to the discrete nature of the data, can be estimated to be $\leq 1 \%$. The importance in observing the propagating front in the Taylor-Couette system for a sufficiently large distance ( $\doteq$ time) is stressed a second time by Fig. 2. From the data shown in Fig. 1, the dimensionless velocities are determined for differently centered time intervals and attributed to the mean of the time interval. At larger times

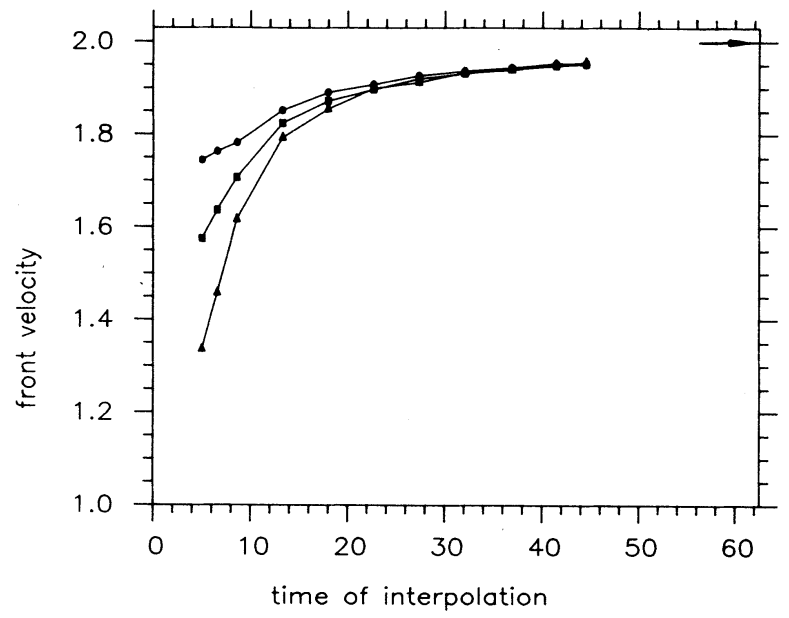

FIG. 2. Dimensionless front velocity $\bar{v}$ obtained by leastsquares interpolation using the data over intervals of $\sim 11\left(d^{2} / v\right)$ centered at different times $t$. The arrow marks the expected theoretical value $\bar{v}=2$. With increasing time of interpolation $t$, the velocities of the different front positions (circles $10 \%$, squares $25 \%$, and triangles $50 \%$ of $u_{\text {max }}$ ) converge denoting the formation of a stationary front. At smaller times, the highintensity front positions move slowest.

convergence can be clearly seen. Besides this, the velocities $\bar{v}_{10 \%}, \bar{v}_{25 \%}$, and $\bar{v}_{50 \%}$ agree within their errors, as expected. For smaller observation times, there are distinct differences, a clear hint that the shape of the front is still changing. Extrapolating the curves of Fig. 2 to $t \rightarrow \infty$ would yield values between 1.965 and 2.02 , depending on the character of the fit function assumed. However, our values for $\bar{v}$ presented in the remainder of this work are actually observed ones which, from Fig. 2, mark a lower boundary.

The results for the determination of the front velocity are summarized in Fig. 3. Shown are the dimensionless front propagation velocities for different values of the driving. The results of the Rayleigh-Bénard system (marked by squares) that have been included as well are in good quantitative agreement with the theoretically predicted value $\bar{v}=2$. In the Taylor-Couette system, the experimental values of Ahlers and Cannell ${ }^{13}$ are approximately a factor of 2 below $\bar{v}=2$. The numerically obtained data of Lücke et al. ${ }^{14}$ give values of approximately $1.75-1.80$, while the value of Neitzel $^{18}$ is slightly above $\bar{v}=2$. The results of our numerical simulation of the full time-dependent Navier-Stokes equations are marked by the large filled circles in Fig. 3. Compared to all the other data the agreement is excellent (one should remember that these data represent a lower boundary; see Fig. 2). Besides this, the $\epsilon$ dependence of the observed front velocities corresponds to the one expressed by Eq. (2).

This investigation also clarifies why the numerical results obtained in the Taylor-Couette system so $\mathrm{far}^{14,15}$ are below $\bar{v}=2$ : The authors used a traveling distance of only 15(d). At this time the front profile, and so the velocity, is not yet stationary. Their data are in agreement with the present results for this distance in Fig. 2. Besides this, the 


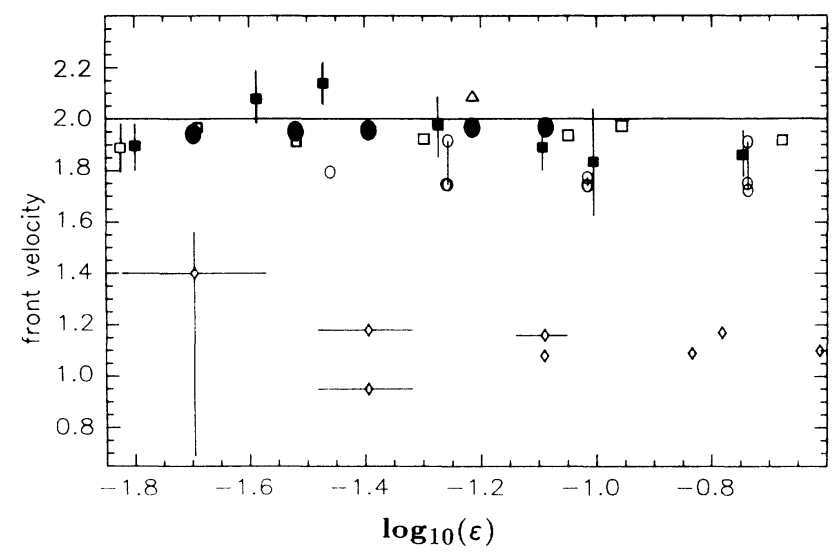

FIG. 3. Dimensionless front velocities as a function of the driving $\epsilon$. We compare results of the present calculations (a) for Taylor-vortex fronts as a function of $\epsilon=T / T_{c}-1$ with those of Ref. $14(0)$, Ref. $18(\triangle)$, and the experiments of Ref. 13 $(\diamond)$. Also shown are experimental results of Ref. $12(\square)$ and numerical results of Ref. 11 ( $\square$ ) for convective fronts in the Rayleigh-Bénard system, as a function of $\epsilon=N_{R_{a}} / N_{R_{a}}-1$. In the present simulation and that of Refs. 11 and $14, \epsilon=0$ marks the critical driving of the respective numerical simulation which is slightly smaller (Refs. 14 and 17) than the theoretical one.

present investigation also very strongly suggests the conjecture that the experimentally observed fronts of Ahlers and Cannell are not yet stationary at a distance of $15(\mathrm{~d})$ from the end plate. In these experiments, observations could be made only for $t<13\left(d^{2} / v\right)$. For longer times, inhomogeneous nucleation intervened. ${ }^{13}$ For $t<13\left(d^{2}\right)$ $v$ ), our numerical results are compatible with the measurements within the uncertainty of the experimental results. We also mention that the slow early-stage evolution of the front in our simulation of the full Navier-Stokes equation differs in two important points from the transients found ${ }^{13}$ for the Ginzburg-Landau equation: Initial transients there are short lived and seem to yield an apparent front speed greater than $\bar{v}=2$, in contrast to the full Navier-Stokes system.

In the Rayleigh-Bénard system a traveling distance of about $20(d)$ seems to be sufficient to determine the front velocity accurately enough. There convection is started locally and instantaneously by a heat pulse ${ }^{11,12,14}$ through a sidewall. In the Taylor-Couette system, however, the initial Ekman vortices have an intensity profile with a finite, exponentially decaying extension. ${ }^{20-22}$ These differences in the starting conditions of the fronts might be responsible for the fact that in the Taylor-Couette system, fronts require a longer time to reach the final propagation speed. However, the front velocity in the Taylor-Couette system can be determined numerically with high precision and is found to be in excellent agreement with the theoretical predictions. Based on this experience we have determined the vortex front propagation velocities also in magnetic
TABLE I. Front velocities in magnetic fluids at $\epsilon=0.0404$ $\left(N_{\mathrm{Re}}=1.02 N_{\mathrm{Re}_{c}}\right)$ for differently oriented fields. The dimensionless velocity $\bar{v}=v /\left(\xi_{0} \sigma_{0} \sqrt{\epsilon}\right)$ deviates from $\bar{v}=2$ by $\leq 3 \%$, thus the description by the amplitude equation is very good. ( $v$ is given in units of $v / d$.)

\begin{tabular}{lccc}
\hline \hline $\begin{array}{l}\text { Orientation of } \\
\text { magnetic field }\end{array}$ & \multicolumn{2}{c}{ Front velocity $v$} \\
& $2 \sqrt{\epsilon} \xi_{0} \sigma_{0}$ & Simulation & $\bar{v}$ \\
\hline$\ldots$ & 2.013 & 1.965 & 1.953 \\
Axial & 3.091 & 3.049 & 1.972 \\
Radial & 3.265 & 3.195 & 1.957 \\
Azimuthal & 3.006 & 2.915 & 1.940 \\
\hline \hline
\end{tabular}

fluids instead of the normal liquid in the gap.

Magnetic fluids are suspensions of small magnetic particles of typical size $10 \mathrm{~nm}$. Each particle is coated by some surfactant to prevent agglomeration and sedimentation. In a magnetic field, the free rotation of the magnetic particles, due to the local vorticity of the suspension, is hindered by their tendency to align parallel to the magnetic field. This gives rise to an additional anisotropic "rotational viscosity $v_{r}$," leading to additional terms in the momentum-balance equation for magnetic fluids. Details on magnetic fluids may be found in Ref. 23.

Here, we investigate the influence of differently orientated magnetic fields on the front propagation velocity in a magnetic fluid in the Taylor-Couette system. Details on the hydrodynamic equations and on the influence of magnetic fields on the critical Taylor number and wave vector may be found in Ref. 16. Therein, all the information on the magnetic field strength and on the specific properties of the magnetic fluid is collected in one parameter, $S=v_{r} / v$. For example, for $S=1$ it is found ${ }^{17}$ that axially, azimuthally, and radially oriented magnetic fields change the values of the parameters $\xi_{0}$ and $\sigma_{0}$ drastically, thus from Eq. (2), the observable front velocities should change too. Assuming $\bar{v}=2$, the theoretically expected values are tabulated in the first row of Table $I$ for a few representative parameter and field configurations. The front velocities actually observed in computer simulation of the proper field equations, corresponding to the magnetic field under consideration, are in very good agreement with these theoretical predictions. The general increase of the front velocity in a magnetic fluid may be understood by an increased coupling due to the additional anisotropic rotational viscosity. On the other hand, $v$ may be transformed, according to Eq. (2), to a dimensionless quantity $\bar{v}=v /\left(\xi_{0} \sigma_{0} \sqrt{\epsilon}\right)$. A comparison of these data (third row in Table I) with $\bar{v}=2$ shows that the deviations are $\leq 3 \%$ and always negative. This is compatible with the findings in Fig. 2. In summary, one also has to conclude that in magnetic fluids vortex front propagation can be described by Eq. (1) and that the agreement is excellent. 


\section{RAPID COMMUNICATIONS}

*Permanent address: Institut für Theoretische Physik, Universität des Saarlandes, D-6600 Saarbrücken, West Germany.

${ }^{1}$ Cellular Structures in Instabilities, edited by J. E. Wesfreid and S. Zaleski (Springer, New York, 1984).

${ }^{2}$ W. van Saarloos, Phys. Rev. A 37, 211 (1988).

${ }^{3}$ G. Dee and J. S. Langer, Phys. Rev. Lett. 50, 383 (1983).

${ }^{4}$ G. Dee, J. Stat. Phys. 39, 705 (1985).

${ }^{5}$ E. Ben Jakob, H. R. Brand, G. Dee, L. Kramer, and J. S. Langer, Physica D 14, 348 (1985).

${ }^{6}$ A. C. Newell and J. A. Whitehead, J. Fluid Mech. 38, 279 (1969).

${ }^{7}$ L. A. Segel, J. Fluid Mech. 38, 203 (1969).

${ }^{8}$ A. C. Newell, in Propagation in Systems Far from Equilibrium, edited by J. E. Wesfreid et al., Springer Series in Synergetics, Vol. 41 (Springer, New York, 1988), p. 122.

${ }^{9}$ A. Kolmogorov, I. Petrovsky, and N. Pisconov, Bull. Univ. Moscow Ser. Int. Sec. A 1, 1 (1937).

${ }^{10}$ D. G. Aronson and H. F. Weinberger, Adv. Math. 30, 33 (1978).

${ }^{11}$ M. Lücke, M. Mihelcic, and B. Kowalski, Phys. Rev. A 35, 4001 (1987).

12J. Fineberg and V. Steinberg, Phys. Rev. Lett. 58, 1332 (1987).
${ }^{13}$ G. Ahlers and D. S. Cannell, Phys. Rev. Lett. 50, 1583 (1983).

${ }^{14}$ M. Lücke, M. Mihelcic, B. Kowalski, and K. Wingerath, in The Physics of Structure Formation: Theory and Simulation, edited by W. Güttinger and G. Dangelmayr, Springer Series in Synergetics, Vol. 37 (Springer, New York, 1987).

${ }^{15}$ M. Lücke, M. Mihelcic, and K. Wingerath, Phys. Rev. Lett. 52, 396 (1985); Phys. Rev. A 31, 396 (1985). In this work $\epsilon_{0}=N_{\mathrm{Re}} / N_{\mathrm{Re}}$ (theory) -1 is defined relative to the theoretical critical Reynolds number.

${ }^{16}$ M. Niklas, Z. Phys. B 68, 493 (1987).

${ }^{17}$ M. Niklas, Kernforschungsanlage Jülich Report No. Jül-2246, 1988 (unpublished); Ph.D. thesis, Rheinisch-Westfälische Technische Hochschule Aachen, 1988.

${ }^{18}$ G. P. Neitzel, J. Fluid Mech. 141, 51 (1984).

${ }^{19}$ M. A. Dominguez-Lerma, G. Ahlers, and D. S. Cannell, Phys. Fluids 27, 856 (1984).

${ }^{20}$ G. Ahlers, D. S. Cannell, M. A. Dominguez-Lerma, and R. Heinrichs, Physica D 23, 202 (1988).

${ }^{21}$ P. Hall, Proc. R. Soc. London, Ser. A 372, 317 (1980).

${ }^{22}$ R. Graham and J. A. Domaradzki, Phys. Rev. A 26, 1572 (1982).

${ }^{23}$ R. E. Rosensweig, in Ferrohydrodynamics, (Cambridge Univ. Press, Cambridge, 1985). 